\title{
Determinação da imunogenicidade de proteínas de amostra de Mycobacterium bovis isolada de bovinos no estado de Goiás, Brasil
}

\author{
Immunogenicity evaluation of proteins from a Mycobacterium bovis isolated from cattle in \\ the State of Goiás, Brasil
}

\section{Cinthya Marques FORTES ${ }^{1}$; Bruno Souza MARQUES'; Viviane Cambuí Mesquita ROCHA²; Ana Paula JUNQUEIRA-KIPNIS ${ }^{1}$}

\author{
${ }^{1}$ Instituto de Patologia Tropical e Saúde Pública da Universidade Federal de Goiás, Goiânia-GO, Brasil \\ ${ }^{2}$ Departamento de Medicina Veterinária Preventiva e Saúde Animal da Faculdade de Medicina Veterinária e Zootecnia da \\ Universidade de São Paulo, São Paulo-SP, Brasil
}

\begin{abstract}
Resumo
A tuberculose bovina persiste em vários países como ameaça à saúde dos rebanhos apesar dos esforços dispensados ao controle e erradicação da doença. O objetivo deste trabalho foi elucidar quais seriam as proteínas mais imunogênicas e, portanto, com potencial de serem utilizadas em novos testes de diagnóstico bovino da tuberculose. Amostras de soro obtidas de animais reatores e não reatores ao teste de tuberculinização intradérmica foram avaliados quanto ao reconhecimento de antígenos proteicos de Mycobacterium bovis, isolado de bovino no Estado de Goiás. A imunogenicidade das proteínas de M. bovis foi obtida por meio da técnica de Western blot. A maioria dos bovinos PPD positivos $(67,92 \%)$ reconheceu o antígeno de $26 \mathrm{kDa}$, sugerindo o seu uso potencial no desenvolvimento de um ensaio sorológico para a tuberculose bovina.
\end{abstract}

Palavras-chave: Tuberculose bovina. Diagnóstico. Proteínas.

\begin{abstract}
Bovine tuberculosis, persists in several countries as a threat to health of the herds despite the efforts given to the control and eradication of the disease. In order to detect immunogenic proteins with a potential to be used in a bovine tuberculosis diagnosis test, serum samples obtained from reactors and no reactors to intradermal tuberculin test, were evaluated for reactivity to Mycobacterium bovis antigens from a bacilli originated in Goiás - Brazil. The proteins immunogenicity from $M$. bovis was obtained by Western blot according to the molecular weight profile. The majority of the PPD positive bovine (67.92\%) recognized a protein with $26 \mathrm{kDa}$, suggesting the use of this protein in a serological test for bovine tuberculosis.
\end{abstract}

Keywords: Bovine tuberculosis. Diagnostic. Proteins.

\section{Introdução}

O Mycobacterium bovis, agente etiológico da tuberculose nos bovinos, provoca perdas econômicas consideráveis vinculadas à restrição ao comércio dos produtos agropecuários e ao custo dispensado a implementação de programas de erradicação da doença em várias partes do mundo ${ }^{1}$.

A estratégia atual de controle da enfermidade baseada no teste de tuberculinização intradérmica preconizada pela Organização Mundial de Saúde Animal (OIE) tem apresentado falhas em detectar todos os animais doentes, por isso, esforços são feitos no senti- do de se encontrar novas formas de diagnóstico para a doença nos bovinos. Apesar de estudos mostrarem uma relação inversa entre a imunidade celular e humoral durante a progressão da doença $\mathrm{a}^{2,3}$, testes de diagnóstico baseado em anticorpos têm sido ampla-

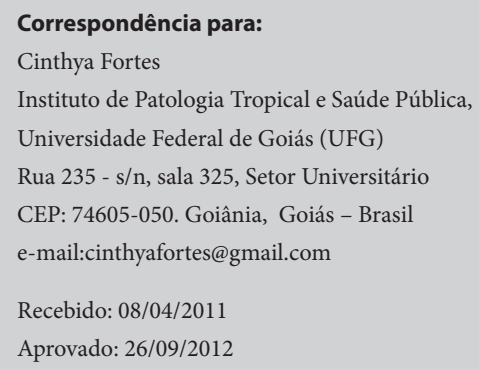


mente pesquisados, principalmente por auxiliar na detecção de animais negativos ao teste de tuberculinização intradérmica com derivado proteico purificado (PPD negativos) ou anérgicos devido ao estágio da doença.

Lyashchenko et al. ${ }^{4}$ afirmaram que ensaios sorológicos seriam úteis ao diagnóstico da tuberculose por serem geralmente simples, rápidos e de custo reduzido. Entretanto, para que fossem eficazes, seria necessária a utilização de antígenos estritamente relacionados ao agente etiológico a fim de melhorar a especificidade do ensaio.

Visando contribuir com o controle da doença nos rebanhos, o presente estudo focou-se na identificação de proteínas imunogênicas de Mycobacterium bovis isolados no estado de Goiás - Brasil, com potencial de serem utilizadas em pesquisa de diagnóstico sorológico da tuberculose bovina.

\section{Material e Método}

O M. bovis foi obtido a partir de linfonodos subescapular e submandibular de quatro bovinos eutanasiados e autopsiados a campo após diagnóstico positivo ao teste intradérmico. $\mathrm{O}$ material foi processado, homogeneizado, suspendido, centrifugado e descontaminado conforme o método de Petroff 5 . Foi semeado em meio Stonebrink a $37^{\circ} \mathrm{C}$ e o crescimento foi observado a partir de 21 dias.

A técnica de extração do DNA foi realizada conforme citado por Van Embden et al. ${ }^{6}$. Para extração do DNA genômico, as amostras das quatro culturas foram colocadas em tubo.

A técnica foi realizada conforme descrito por Kamerbeek et al. ${ }^{7}$ Com reações de $50 \mu \mathrm{L}$, contendo água ultra pura, tampão de reação, dNTP $(1,25 \mathrm{mM})$, 10pmol de cada oligonucleotídeo, 50mM de $\mathrm{MgCl}$, $10 \mathrm{mM}$ de Tris- $\mathrm{HCl}$ (pH 8,3), iniciador (DRa 5'GGGTTTGGGTCTGACGAC-3' biotinado em DRb 5'-CCGAGAGGGGACGGAAAC -3'), TAQ polime- rase (1,25 unidades) e1 $\mu \mathrm{L}$ DNA. O ciclo empregado na PCR foi: desnaturação a $96^{\circ} \mathrm{C}$ por 3 min., 30 vezes do ciclo de amplificação, $96{ }^{\circ} \mathrm{C}$ por $1 \mathrm{~min}$., $55^{\circ} \mathrm{C}$ por $1 \mathrm{~min}$. e $72{ }^{\circ} \mathrm{C}$ por 30 seg., seguido de um ciclo de extensão final de $72{ }^{\circ} \mathrm{C}$ por $10 \mathrm{~min}$. Após a amplificação, $20 \mu \mathrm{L}$ do produto de PCR foi adicionado em $170 \mu \mathrm{L}$ de $2 \mathrm{X}$ SSPE/0,1\% SDS (20X SSPE: 0,2M $\mathrm{NaHPO}_{4}, 3,6 \mathrm{M}$ de $\mathrm{NaCl}, 20 \mathrm{Mm}$ de EDTA), feita a desnaturação a $99^{\circ} \mathrm{C}$ por $15 \mathrm{~min}$. e colocado imediatamente em gelo para se evitar a reassociação das fitas. A membrana foi então sensibilizada lavando-a com 2 X SSPE $0,1 \%$ SDS a $60{ }^{\circ} \mathrm{C}$ durante 5 min. e ajustada no miniblotter de modo a ficar com a linha de aplicação das sondas perpendicular a das amostras. Após a aplicação das amostras, foi feita a hibridização por $60 \mathrm{~min}$. a $60{ }^{\circ} \mathrm{C}$. Sequencialmente, a membrana foi lavada por duas vezes com 2 X SSPE/0,5\% SDS/10 min. a $60^{\circ} \mathrm{C}$, resfriada em uma bolsa de hibridização e incubada com conjugado streptavidina-peroxidase diluída em 2X SSPE/0,5\% (etapa realizada a $42{ }^{\circ} \mathrm{C} / 60$ minutos). Novamente a membrana foi lavada duas vezes em $2 \mathrm{X}$ SSPE/0,5\% SDS/10 min. a $42{ }^{\circ} \mathrm{C}$ seguido de mais duas lavagens em temperatura ambiente com 2XSSPE, após, a membrana foi incubada com reagentes de quimioluminescência/ 5 min. (ECL ${ }^{\circledR}$ Amersham Pharmacia). A documentação foi realizada em filme radiográfico e a revelação com reagentes para radiografia, seguindo a indicação do fabricante. Os controles positivos usados foram DNA cromossomal de $M$. tuberculosis $\mathrm{H} 37 \mathrm{Rv}$ e $M$. bovis BCG.

A análise dos espoligotipos foi baseada no código binário obtido conforme catalogado no banco de dados do site mbovis.org.

As bactérias foram raspadas do meio de cultura e maceradas em nitrogênio líquido até a obtenção de um pó fino. O produto foi transferido para um tubo falcon contendo $2 \mathrm{~mL}$ de Tris-EDTA $20 \mathrm{mM}$, com os inibidores de protease TLCK (Sigma Aldrich, St Louis, EUA), PMSF (Sigma Aldrich, St Louis, EUA) $(20 \mu \mathrm{L}$ de cada) e alfafenotroína $(60 \mu \mathrm{L})$ e, em seguida, 
homogeneizado. A mistura antigênica foi aliquotada $(800 \mu \mathrm{L} /$ tubo), acrescida de tampão de amostra contendo 2 -mercaptoethanol $(200 \mu \mathrm{L} /$ tubo $)$ e inativadas a $85^{\circ} \mathrm{C} / 20 \mathrm{~min}$., em seguida, conservadas à temperatura de $-20^{\circ} \mathrm{C}$.

A eletroforese em gel de poliacrilamida (SDS-PAGE) foi realizada sob condições redutoras como citado por Laemmli $^{8}$. Os géis foram preparados com uma solução de acrilamida (Sigma Aldrich, St Louis, EUA), Bis-acrilamida (Sigma Aldrich, St Louis, EUA), água destilada e solução Trizma-base $0,75 \mathrm{M} \mathrm{pH} 8,8$, para gel de corrida e Trizma 0,25 M pH 6,8 para gel de empilhamento. Após o preparo dos géis, uma alíquota do extrato antigênico total foi centrifugada por $10 \mathrm{~min}$. a $6000 \mathrm{rpm}$ e, em seguida, aquecida a $96^{\circ} \mathrm{C} / 5 \mathrm{~min}$., e $150 \mu \mathrm{L}$ do sobrenadante da amostra foi distribuído nas canaletas do gel de empilhamento. A corrida ocorreu sob um campo elétrico de 70 volts até a entrada das amostras no gel de corrida e, em seguida, o campo elétrico utilizado foi de 100 volts. Foi utilizado padrão dual color de peso molecular (Precision Plus Protein, Bio Rad, USA) durante a corrida que foi realizada em duplicata, sendo que um gel foi corado com azul de comassie e o outro transferido para a membrana de nitrocelulose. A amostra antigênica fracionada foi então transferida para a membrana de nitrocelulose como descrito por Towbin, Staehelin e Gordon' ${ }^{\text {. A efici- }}$ ência da transferência foi visualizada pelo uso do corante Ponceau Red. As tiras foram bloqueadas com solução de leite em pó desnatado à temperatura ambiente e incubadas com amostras dos soros dos bovinos na diluição 1:100 em solução de bloqueio $2 \%$ por 2 horas a $37^{\circ} \mathrm{C}$. Após a lavagem em solução PBS tween 20 a 0,05\%, as tiras foram incubadas com anticorpo secundário conjugado-peroxidase anti-IgG bovino (Jackson Immuno Reserch Laboratories, West Groove, USA) em solução bloqueadora por 1 hora na proporção 1:5000 e, posteriormente, reveladas.

Foi utilizado um painel de 93 amostras de soro bovino, sendo 53 provenientes de animais PPD positivos de fazendas localizadas no estado de Goiás, Distrito
Federal e Minas Gerais, e 40 provenientes de animais PPD negativos, sendo qu e 20 amostras deste último grupo vieram de região livre da tuberculose dos Estados Unidos da Améerica, gentilmente cedidas pelo Dr. Ray Waters, USDA, e as outras 20, da mesma região do grupo dos PPD positivos. As outras amostras de soro dos bovinos brasileiros foram enviadas pela Empresa Brasileira de Pesquisa Agropecuária - Recursos Genéticos e Biotecnologia (EMBRAPA-CENARGEN) e mantido o sigilo quanto às propriedades rurais de origem. Todos os animais foram submetidos à prova de PPD comparada para que fossem considerados positivos ou negativos.

\section{Resultados}

A revelação radiográfica da membrana de Spoligotyping das quatro cepas analisadas evidenciou mesmo padrão de hibridização com ausência dos espaçadores 39 a 43, característica de todas as cepas de Mycobacterium bovis, e código binário 1100000101111110111 101111000011111111100000 que possibilitou a identificação das cepas no site mbovis.org como sendo do espoligotipo SB1145. A documentação radiográfica pode ser visualizada na figura 1 . Todas as cepas foram idênticas e, por isso, apenas um isolado foi utilizado para a caracterização da imunogenecidade.

Três proteínas se mostraram mais evidentes no gel de poliacrilamida, tendo aproximadamente 16, 26 e $40 \mathrm{kDa}$ (Figura 2).

O soro de bovinos PPD positivos identificou proteínas antigênicas diferentes das evidenciadas em SDS-PAGE e embora o padrão de reconhecimento desses antígenos tenha sido variável com o reconhecimento das proteínas de $16,19,26,37,50,55$, e $70 \mathrm{kDa}$, duas destas foram regularmente detectadas, que são as de massa molecular de 19 e $26 \mathrm{kDa}$, sendo que a de aproximadamente $26 \mathrm{kDa}$, sugestiva de ser a MPB83, foi a mais frequente dentre os antígenos desta pesquisa (Figura 3). 


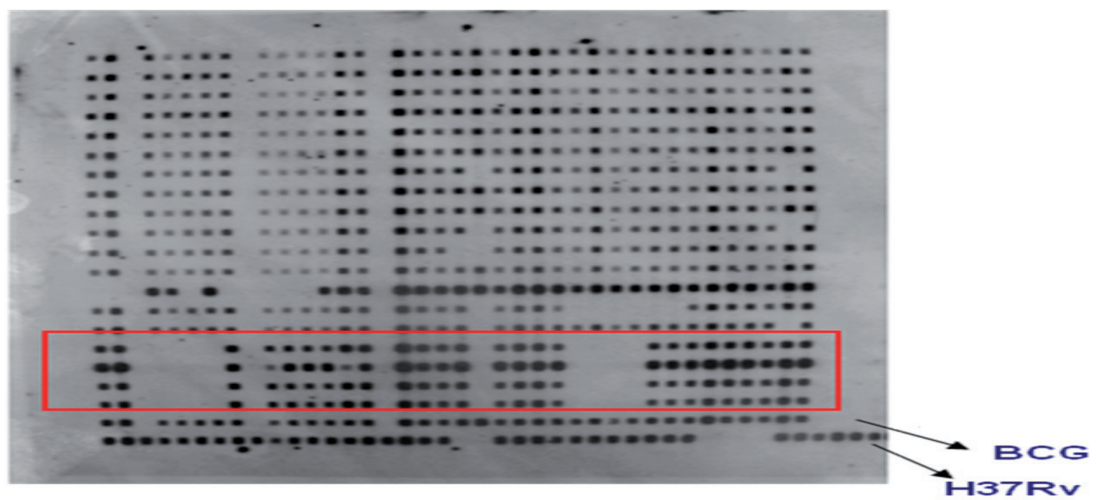

Figura 1 - Documentação radiográfica do padrão de hibridização dos quatro isolados de Mycobacterium bovis

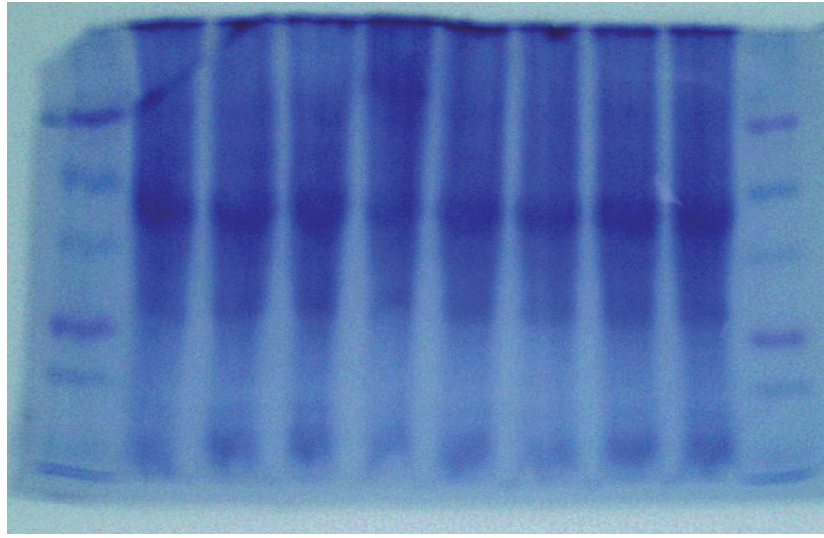

Figura 2 - Gel de poliacrilamida da eletroforese, corado com azul de comassie, evidenciando a separação das proteínas totais de Mycobacterium bovis

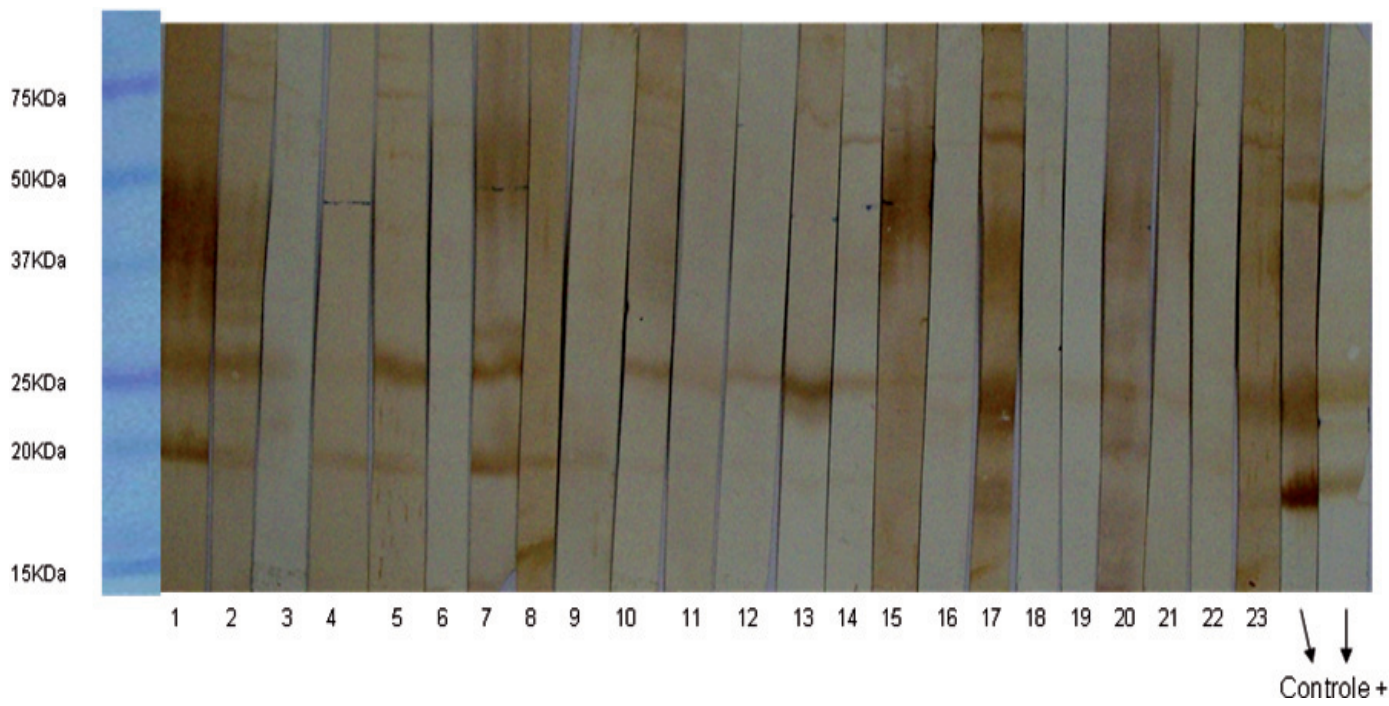

Figura 3 - Imunoblot representativo das amostras dos animais PPD positivos 
O padrão de reconhecimento de antígenos de M.bovis pelos anticorpos ( $\mathrm{IgG}$ ) do soro dos animais PPD negativos avaliados pelo imunoblot identificou a proteína de $35 \mathrm{kDa}$ em cinco $(12,5 \%)$ das amostras testadas e os anticorpos presentes no soro dos animais de região livre de tuberculose (controles negativos) não apresentaram qualquer reatividade contra as proteínas isoladas de M. bovis.

Bandas imunogênicas para as frações proteicas de 16,19 e $37 \mathrm{kDa}$ foram observadas somente nos animais PPD positivos enquanto que as de 35 e $40 \mathrm{kDa}$ foram reconhecidas apenas pelo soro de animais PPD negativos. A frequência das bandas visualizadas entre os dois grupos pode ser visto na tabela 1 .

\section{Discussão}

A caracterização molecular das cepas de M. bovis possibilitou identificar que estas eram pertencentes ao mesmo clone, o que pode ser justificado pelo fato de terem sido isoladas de animais de uma mesma região, o que facilitaria a disseminação desta entre eles, ou até mesmo devido à contaminação durante o cultivo no laboratório. A mesma cepa foi identificada em estudos anteriores por Rosales ${ }^{10}$, no estado de São Paulo - Brasil e por Rodriguez et al. ${ }^{11}$ na região de Castille e Leon na Espanha. Entretanto, maiores estudos são necessários a fim de que se conheça a origem desta cepa SB1145.

Pesquisas anteriores também mostraram que a resposta humoral é variável e acompanha o processo evolutivo da infecção ${ }^{12,13}$ o que justificaria os diferentes padrões de bandas antigênicas encontradas em diferentes fases de disseminação da doença ${ }^{14,15}$. Lyashchenko et al. ${ }^{4}$ após analisarem a cinética da resposta de anticorpos contra proteínas específicas, citaram ainda uma variação no padrão de imunodominância de antígenos durante o curso da infecção. Entretanto, foi observado no presente estudo que independentemente de se ter conhecimento sobre o

\begin{tabular}{|c|c|c|}
\hline Bandas & PPD + * & PPD - ** \\
\hline$\approx 16 \mathrm{kDa}$ & $10(18,86)$ & 0 \\
\hline$\approx 19 \mathrm{kDa}$ & $20(37,73)$ & 0 \\
\hline$\approx 26 \mathrm{kDa}$ & $36(67,92)$ & $2(5)$ \\
\hline$\approx 35 \mathrm{kDa}$ & 0 & $5(12,5)$ \\
\hline$\approx 37 \mathrm{kDa}$ & $5(9,43)$ & 0 \\
\hline$\approx 40 \mathrm{kDa}$ & 0 & $2(5)$ \\
\hline$\approx 50 \mathrm{kDa}$ & $10(18,86)$ & $2(5)$ \\
\hline$\approx 55 \mathrm{kDa}$ & $8(15,09)$ & $1(2,5)$ \\
\hline$\approx 70 \mathrm{kDa}$ & $5(9,43)$ & $1(2,5)$ \\
\hline
\end{tabular}

Total de 53 animais avaliados, ${ }^{* *}$ total de 40 animais avaliados

estágio ou a via de infecção dos animais positivos ao teste intradérmico, a maioria $(67,92 \%)$ destes, apresentaram sororeatividade contra a proteína de $26 \mathrm{kDa}$ (MPB83).

Recentemente, pesquisas têm utilizado a proteína recombinante MPB83 em ensaios que combinam diferentes antígenos como, por exemplo, o MAPIA (Imunoensaio de Múltiplos Antígenos Impressos) e o ensaio de fluxo lateral, confirmando o MPB83 como um dos mais promissores antígenos para sorodiagnóstico da tuberculose não só em bovinos, mas também em vários animais silvestres ${ }^{16,17}$.

A segunda proteína mais reconhecida pelos animais PPD positivos no presente estudo foi a de $19 \mathrm{kDa}$, sugerindo que esta imunogenicidade apresentada seja resultante de reação cruzada com micobactérias não tuberculosas, uma vez que trabalhos anteriores como o de Huntley, Stabel e Bannantin ${ }^{18}$, por exemplo, citam este antígeno como sendo altamente expresso por Mycobacterium avim subsp. paratuberculosis causador de enterite crônica conhecida como paratuberculose ou doença de Johne nos bovinos. Da mesma forma, a proteína de $35 \mathrm{kDa}$ dos PPD negativos, também é amplamente estudada em rebanhos com paratuberculose e tem sido citada como importante fator de virulência no início desta infecção. 


\section{Conclusão}

Os resultados encontrados no presente estudo confirmam o potencial da proteína de $26 \mathrm{kDa}$ (MPB83) de Mycobacterium bovis no desenvolvimento de ensaios para diagnóstico sorológico para a tuberculose bovina, pois ficou demonstrada a imunogenicidade desta proteína também entre os bovinos naturalmen-

\section{Referências}

1. MCNAIR, J.; CORBETT, D. M.; GIRVIN, R. M.; MACKIE, D. P.; POLLOCK, J. M. Characterization of the early antibody response in bovine tuberculosis: MPB83 is a early target with diagnostic potential. Scandinavian Journal of Immunology, v. 53 , n. 4 , p. $365-371,2001$.

2. KAUFFMAN, S. H. How can immunology contribute to the control of tuberculosis? Nature Reviews Immunology, London, v. 1, n. 1, p. 20-30, 2001.

3. WELSH, D. M.; CUNNINGHAM, R. T.; CORBETT, R. M. G.; MCNAIR, J.; SKUCE, R. A.; BRYSON, D. G.; POLLOCK, J. M. Influence of pathological progression on the balance between cellular and humoral immune responses in bovine tuberculosis. Immunology, v. 114, n. 1, p. 101-111, 2005.

4. LYASHCHENKO, K. P.; POLLOCK, J. M.; COLANGELI, R.; GENNARO, M. L. Diversity of antigen recognition by serum antibodies in experimental bovine tuberculosis. Infection and Immunity, Washington, v. 66, n. 11, p. 5344-5349, 1998.

5. VESTAL, A. L. Procedures for isolation and identification of Mycobacteria. Atlanta, Georgia: U.S.Departament of health, Education and Welfare; Centers for Diseases Control and Prevention, 1977. p. 15-19. (Publication $\mathrm{n}^{\circ}$ CDC-77-8230).

6. VAN EMBDEN, J. D.; CAVE, M. D.; CRAWFORD, J. T.; DALE, J. W.; EISENACH, K. D.; GICQUEL, B.; HERMANS, P.; MARTIN, C.; MCADAM, R.; SHINNICK, T. M. Strain identification of Mycobacterium tuberculosis by DNA fingerprinting: recommendations for a standardized methodology. Journal of Clinical Microbiology, v. 31, n. 2, p. 907-914, 1993.

7. KAMERBEEK, J.; SCHOULS, L.; KOL, A.; AGTERVELD, M. V.; SOOLINGEN, D. V.; KUIJPER, S.; BUNSCHOTEN, A.; MOLHUIZEN, H.; SHAW, R.; GOYAL, M.; EMBDEM, J. D. Simultaneous detection and strain differentiation of Mycobacterium tuberculosis for diagnosis and epidemiology. Journal of Clinical Microbiology, v. 35, n. 4, p. 907-914, 1997.

8. LAEMMLI, U. K. Cleavage of structural proteins during the assembly of the head of bacteriofage T4. Reprinted from Nature, v. 227, n. 5259, p. 680-685, 1970.

9. TOWBIN, H.; STAEHELIN, T.; GORDON, J. Eletroforetic transfer of proteins from polyacrylamide gels to nitrocellulose sheets: Procedure and some applications. Proceedings of the National Academy of Sciences of the USA (PNAS), v. 76, p. 4350-4354, 1979.

10.ROSALES C. A. R. Sistema de detecção de focos de tuberculose bovina no Estado de São Paulo utilizando métodos moleculares e epidemiológicos [online]. 2005. $86 \mathrm{f}$. Tese (Doutorado em Epidemiologia Experimental e Aplicada às Zoonoses) - Faculdade de Medicina Veterinária e Zootecnia, te infectados e não só entre os experimentalmente infectados, como citado por pesquisas anteriores.

\section{Agradecimentos}

Ao Instituto de Patologia Tropical e Saúde Pública (IPTSP/UFG); à Universidade de São Paulo (USP) e à Empresa Brasileira de Pesquisa Agropecuária (EMBRAPA-CENARGEN).

Universidade de São Paulo, São Paulo, 2005. Disponível em: $<$ http://www.teses.usp.br/teses/disponíveis/10/10134/tde25102006-154614/publico/CesarAlejandroRosalesRodrigues. pdf>. Acesso em: 20 set. 2009.

11.RODRÍGUEZ, S.; ROMERO, B.; BEZOS, J.; DE JUAN, L.; ÁlVAREZ, J.; CASTELLANOS, E.; MOYA, N.; LOZANO, F.; GONZÁLEZ, S.; SÁEZ-LLORENTE, J. L.; MATEOS, A.; DOMÍNGUEZ, L.; ARANAZ, A. 2009. High spoligotype diversity within a Mycobacterium bovis population: Clues to understanding the demography of the pathogen in Europe. Veterinary Microbiology, v. 141, n. 1/2, p. 89-95. [online]. Disponível em: <http://www.ncbi.nlm.nih.gov/ pubmed/19720476>. Acesso em: 20 nov. 2009.

12. POLLOCK, J. M.; WELSH, M. D.; MCNAIR, J. Immune responses in bovine tuberculosis: Towards new strategies for the diagnosis and control of disease. Veterinary Immunology and Immunopathology, Amsterdam, v. 108, n. 1/2, p. 37-43, 2005.

13.WATERS, W. R.; PALMER, M. V.; THACKER, T. C.; BANNANTINE, J. P.; VORDERMEIER, H. M.; HEWINSON, R. G.; GREENWALD, R.; ESFANDIARI, J.; MCNAIR, J.; POLLOCK, J. M.; ANDERSEN, P.; LYASHCHENKO, K. P. Early antibody responses to experimental Mycobacterium bovis infection of cattle. Clinical and Vaccine Immunology, Washington, v. 13, n. 6, p. 648-654, 2006.

14.CATALDI, A.; ROMANO, M. I.; BIGI, F. A western blot characterization of Mycobacterium bovis antigens recognized by cattle será. Research in Microbiology, v. 145, n. 9, p. 689-698, 1994.

15. POLLOCK, J. M.; ANDERSEN, P. Predominant recognition of the ESAT-6 protein in the first phase of infection with Mycobacterium bovis in cattle. Clinical and Diagnostic Laboratory Immunology, v. 65, n. 7, p. 2587-2592, 1997.

16. WHELAN, C.; SHURALEV, E.; O'KEEFFE, G.; HYLAND, P.; KWOK, H. F.; SNODDY, P.; O'BRIEN, A.; CONNOLLY, M.; QUINN, P.; GROLL, M.; WATTERSON, T.; CALL, S.; KENNY, K.; DUIGNAN, A.; HAMILTON, M. J.; BUDDLE, B. M.; JOHNSTON, J. A.; DAVIS, W. C.; OLWILL, S. A.; CLARKE, J. Multiplex Immunoassay for Serological Diagnosis of Mycobacterium bovis Infection in Cattle. Clinical and Vaccine Immunology, v. 15, n. 12, p. 1834-1838, 2008.

17.WIKER, H. G. MPB70 and MPB83 - Major antigens of Mycobacterium bovis. Scandinavian Journal of Immunology, v. 69, p. 192-199, 2009.

18.HUNTLEY, J. F. J.; STABEL, J. R.; BANNANTIN, J. P. 2005. Immunoreactivity of the Mycobacterium avium subsp. Paratuberculosis $19 \mathrm{kDa}$ lipoprotein. [online] BMC Microbiology, v. 5, n. 1, 2005. Disponível em <http://www. biomedcentral.com/1471-2180/5/3>. Acesso em: 5 dez. 2009. 